\title{
Bright Light Induces Mitochondrial Fission in Photoreceptors: Implications in Retinal Degeneration
}

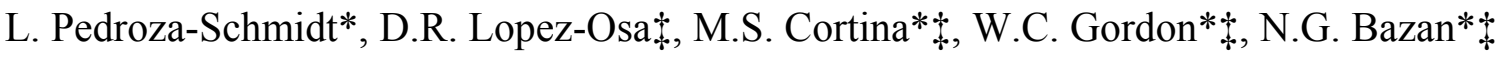 \\ * Department of Ophthalmology, LSU Health Sciences Center, 2020 Gravier St., New Orleans, LA \\ 70112 \\ \$ Neuroscience Center, LSU Health Sciences Center, 2020 Gravier St., New Orleans, LA 70112
}

Exposure to intense light triggers events that lead to apoptosis and retinal cell death. Light-treated rhodopsin -/- rats and normal animals with inhibition of vitamin A reisomerization do not lose photoreceptors, showing that light-mediated photoreceptor death occurs by way of visual pigment [1]. Absorbance of excessive photons leads to oxidative stress, but antioxidant pretreatment preserves photoreceptors, suggesting that mitochondria are involved in the death process. Once begun, rods undergo apoptosis and disappear within 36-48 h [2]. Mitochondrial oxidative stress causes release of pro-apoptotic molecules, induction of enzymatic cascades, DNA fragmentation, and cytoplasmic densification [3], followed by cell loss. However, evidence that some rods recover from light damage [4] led us to examine the process of rod death. Time courses using TUNEL and DNA laddering showed nuclear damage peaking at $24 \mathrm{~h}$ after light [2], and the nuclear repair enzyme DNA polymerase $\beta$ showed a similar up-regulation at $24 \mathrm{~h}$ [3]. However, mitochondrial DNA polymerase $\gamma$ peaked at 6 hours post-stimulus, coinciding with onset of mDNA fragmentation [5], suggesting that early events in photoreceptor apoptosis may involve mitochondria. Finally, it has been shown in vitro with COS-7, HeLa, CHO, yeast cells and others that mitochondrial fragmentation (fission) may be an early event in apoptotic cell death [6].

Rats were dark adapted, light treated for 5 hours in fluorescent light, and returned to darkness. Retinas were collected at various times throughout. Following conventional fixation in glutaraldehyde/formaldehyde and osmium tetroxide, tissue was embedded in an Epon-Araldite mixture, sectioned, and contrasted with lead and uranium salts.

Rats have 8 long peripherally located mitochondria per rod inner segment. Electron micrographs revealed that within 2 hours after light onset, mitochondria had begun to undergo division, forming chains of spherical mitochondria. Some sections showed early division events with complete mitochondrial inner membrane fission within single intact outer membranes. This was observed up to 6 hours following light treatment. Thereafter, rod nuclei became condensed, undergoing blebbing and dissolution characteristic of photoreceptor apoptosis [7]. This is the first time mitochondrial fission has been reported in vivo within neurons, and suggests that maintenance of mitochondria may provide a means of intervention and/or protection from degenerative diseases of the nervous system.

[1] C Grimm et al., Nat Genet. 25 (2000) 63.

[2] WC Gordon et al, Invest Ophthalmol Vis Sci. 43 (2002) 3511.

[3] CE Remé et al., Degenerative Diseases of the Retina. Plenum Press. New York. 1995.

[4] M Moriya et al., Cell Tissue Res. 246 (1986) 607.

[5] MS Cortina et al., Exp Eye Res. in press.

[6] Z Mukamel and A Kimchi. J Biol Chem. 279 (2004) 36732.

[7] WC Gordon and NG Bazan. Biochemistry of the Eye. Chapman \& Hall. London. 1997.

Grant support: NIH/NEI R01 EY05121 and DARPA MDA972-03-C-0100 

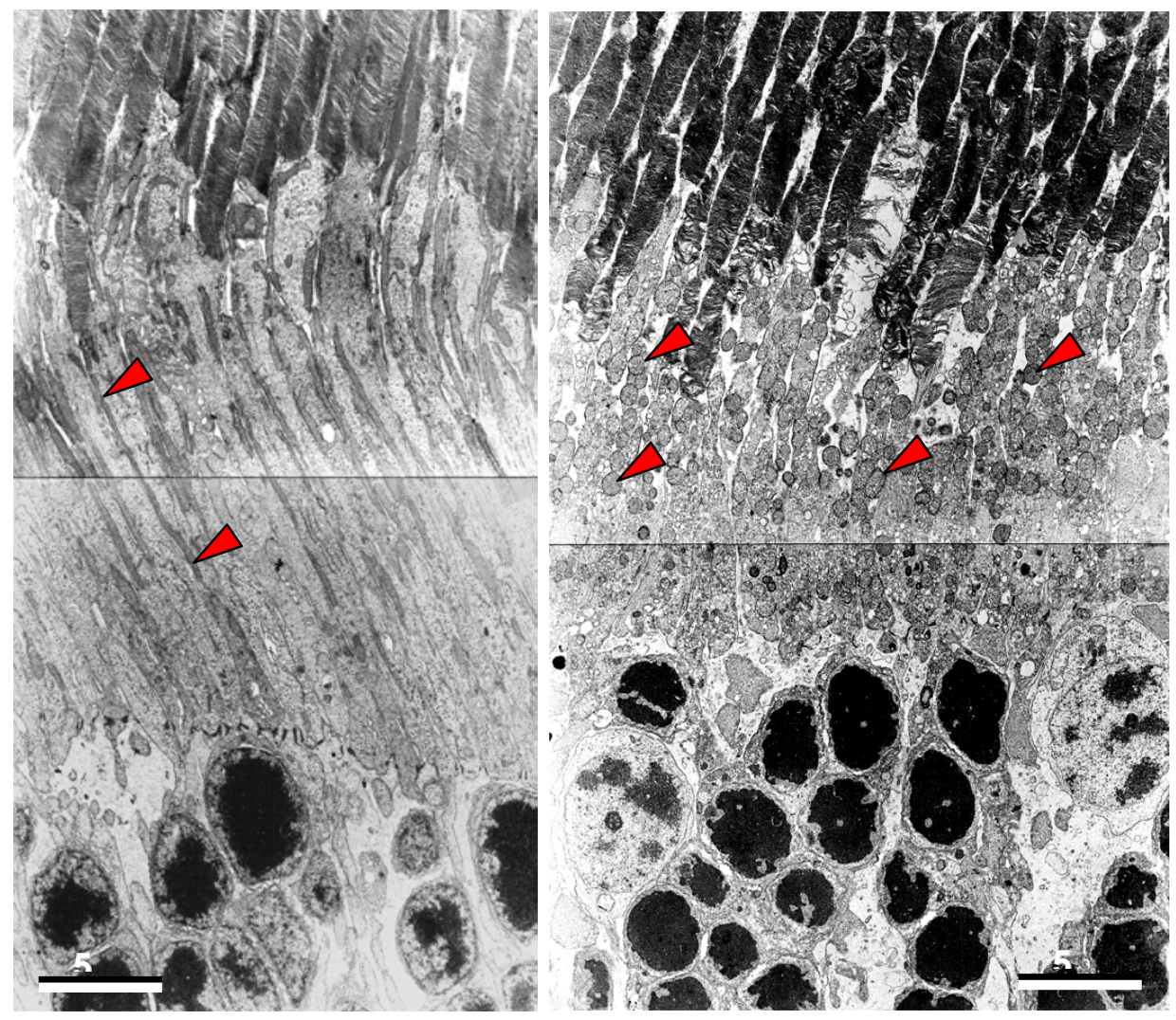

Fig 1. Rat retinas. Normal healthy rod photoreceptors have 8 long peripherally located mitochondria per cell. A single mitochondrion is delineated by the arrows at left. At right is a retina after $5 \mathrm{~h}$ of light $+2 \mathrm{~h}$ of darkness. The arrows point to several mitochondria among hundreds. Both micrographs are shown at the same magnification.
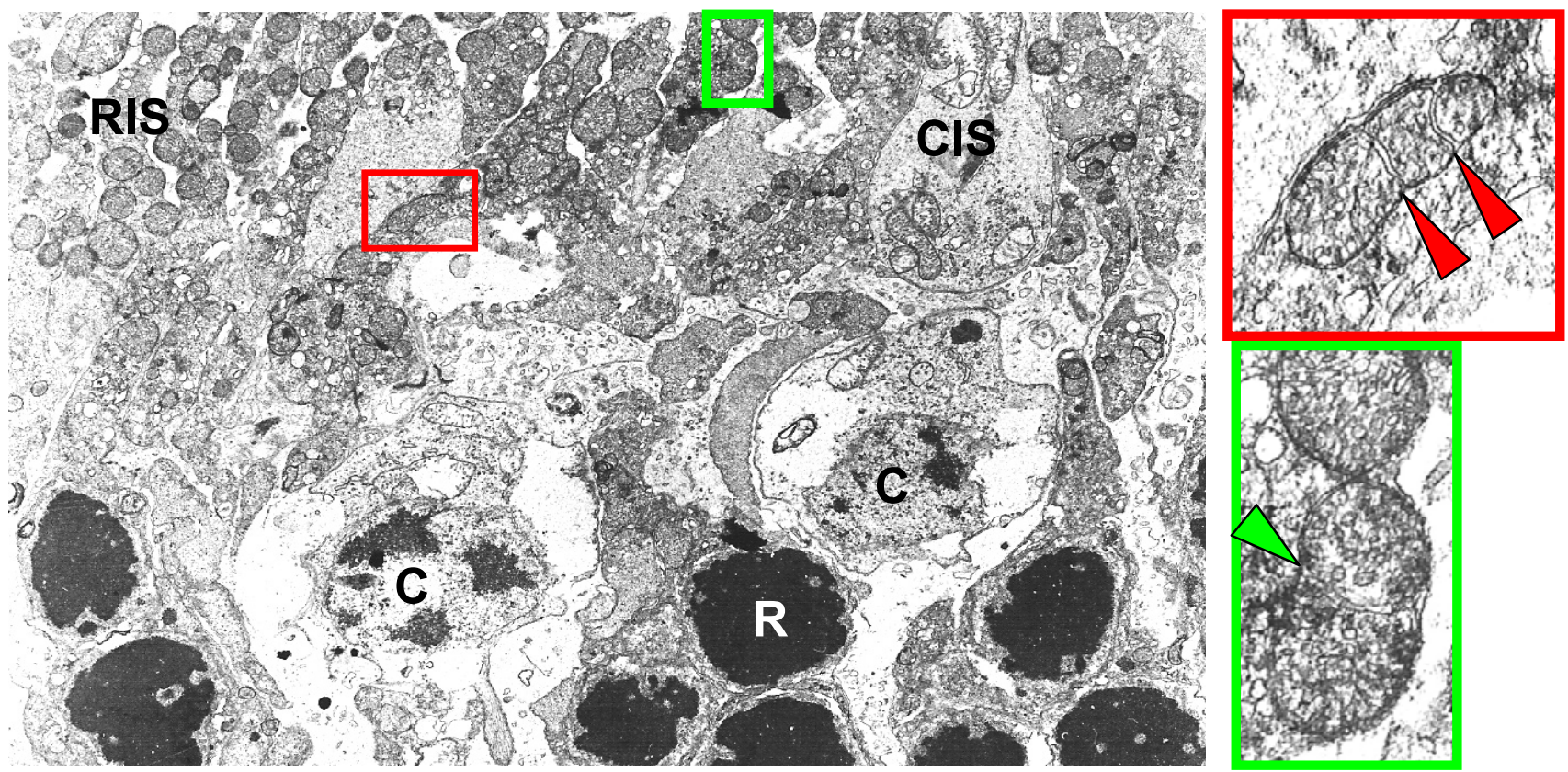

Fig 2. Rat retina viewed at $5 \mathrm{~h}$ light $+3 \mathrm{~h}$ dark. Insets show complete mitochondrial inner membrane fission (arrows). 